\title{
NONCONTACT METHOD OF TEMPERATURE MEASUREMENT BASED ON THE PHENOMENON OF THE LUMINOPHOR TEMPERATURE DECREASING
}

\author{
Vasyl V. Kukharchuk, Valerii F. Hraniak, Yurii G. Vedmitskyi, Volodymyr V. Bohachuk \\ Department of Theoretical electrical engineering and electric measurings, Vinnytsya National \\ Technical University, \\ 95 Khmelnitskoye Shose, Vinnitsya, 21021, Ukraine
}

\begin{abstract}
In the paper is suggested new noncontact method of temperature measurement based on the phenomenon of the luminophor temperature decreasing, is developed the construction of the primary measurement transformer, that implements suggested method, and got its transformation equation.
\end{abstract}

Key words: temperature, luminiscence, luminophor, radiation, measurement.

\section{INTRODUCTION}

Temperature is one of the main parameters that determines chemical and physical characteristics of the material [1-3]. Dielectric characteristics of the winding insulation of the electric machinery depend from temperature as well, this fact determines the necessity to hold to the temperature conditions within certain limits. For defect-free equipment temperature maintenance of the winding insulation within certain limits is realized automatic by providing acceptable exploitation conditions. But, dielectric disturbance of the interturn insulation there is temperature increasing in the defective part that, activating snowballing process, causes to the following thermal breakdown, its speed increases vary with the time of the defect development. On time determination of such defects is especially important for using of the generator sets of the industrial electric generating station [4], where on time determination of the anomalous increasing of the pole windings is an upcoming trend for the avoidance of the emergency situations, which can lead to huge negative effects. Although, widely using of the analogous systems are mainly complicated, on the one hand, by limited capacity of the contact thermometers using for the temperature measurement of the rotable parts of the power-units, on the other hand - with a low accuracy and a sluggish of the noncontact thermometers, that does not allow to determine the defect on the early stages of the development. So, the development of the new highlyaccurate noncontact methods and instruments of temperature measurement is an actual research and practice task, its solution will allow to increase the performance reliability of the power equipment.

\section{TASK DESCRIPTION}

Nowadays the main part of the noncontact instruments of temperature measurement is based on using of the pyrometric techniques of heat metering, their main idea is a photofixation of the thermal emission density of the research object [2]. The main lack of these techniques of the temperature measurement under $180{ }^{\circ} \mathrm{C}$, that is the high limit of the temperature acceptable deviation of the $\mathrm{H}$-level insulation (the most heat-resisting of the power equipment insulation) [5], is insufficient density. So, according to Stefan-Boltzmann Law [6], totally black body within the temperature limits $20-180{ }^{\circ} \mathrm{C}$ will have a full density of the thermal emission within the limits $0,042-0,24 \mathrm{Wt} / \mathrm{sm}^{2}$. Herewith, it is necessary to take into account that for the real physical objects the emissivity factor is always less than one $[7,8]$, and the real pyrometer records density of the thermal emission in limited infrared band of the emission frequency from the surface of the measurement object, the effective area is not more than some $\mathrm{mm}^{2}$ [2]. The attenuation of the thermal emission must be also considered, it takes place during its propagation from the research object to the photodetector, this emission is inversely related to the attenuation coefficient of the propagation medium and the squared distance between the research object and the photodetector $[6,8,9]$. It is obvious that for the real pyrometer the capacity of the thermal emission, which is recorded with the photodetector, will be less than capacity emitting from sm2 of the totally blackbody radiator. This leads to a little responsively of such devices and a significant error that can be added to the measurement result with the receiver noise, as well as outside sources of the infrared radiation, as any material with temperature more than $0^{\circ} \mathrm{K}$.

According to the above, we can make a conclusion, that the main task, developing of the highly-accurate noncontact device for the temperature measurement within the limits of measurement, is the increasing of the responsivity of the informative parameter that transfers information about the temperature of the research object. There is also another large problem limiting the accuracy of the noncontact devices for the temperature measurement, it is using infrared radiation as the informative parameter, as in the main environments of the research object location there is a high density of the electromagnetic waves of this spectral range which appear as a result of the other objects measurement. So, another important task that must be solved during creating of the highly-accurate noncontact devices for temperature measurement is transferring of the informative parameter within the limit of higher 
frequencies in which the influence of the other objects of the environment on the measurement results will be essentially less.

\section{ANALYSES OF THE TASK SOLVING WAYS}

In papers $[7,10,11]$ there is demonstrated that for the number of the luminophors, under radiation excitation of the ultraviolet or X-ray spectrum, functional relation of the luminescence radiation intensity and the luminophor temperature. At the same time, for different luminophors in other limits of the temperature changing under temperature there is increasing [10], and decreasing [7,10,11] of the density of the luminescence radiation. As the maximum of the luminescence radiation of such luminophors usually is in the visible or close ultraviolet region [7, 10, 11], in which the influence of other environment objects on the measurement results is lower, this allows to consider luminescence radiation as the advanced informative parameter for the distant temperature measurement of the luminophor. At the same time, in case of the thermal balance of luminophor and the measurement object (for example, application of the leaf of the luminescent coating on the surface of the pole winding of the electrical machine), this radiating can be an informative parameter of its temperature. From the physical point of view the types of luminophors activity under the temperature change can be explained by different physical phenomenon. In case of increasing of the density of the luminescent radiating under temperature increasing, probably, there is increasing of the luminescent centers number, their own thermal stored energy is enough for moving on the higher energy level under their radiating with a quantum with certain energy. In this case, for luminescent center to take part in the creation of the luminescent radiation, the equation must hold true:

$$
E_{T} \leq E_{q}+E_{t}
$$

where $\mathrm{E}_{T}$ - energy transferring of the luminescent center on the high energy level; $\mathrm{E}_{q}$ - energy of a quantum of the outside radiation, that leads to the luminescent appearing; $\mathrm{E}_{t}$ - their own thermal stored energy of a elementary particle.

At this time there is a phenomenon of the luminophor underexcitation that is evident as decreasing of the quantum yield of luminescence. Confirmation of made hypothesis can be the results of the experimental researches LGBO: Ce - crystal (figure 1), considered in the paper [10].

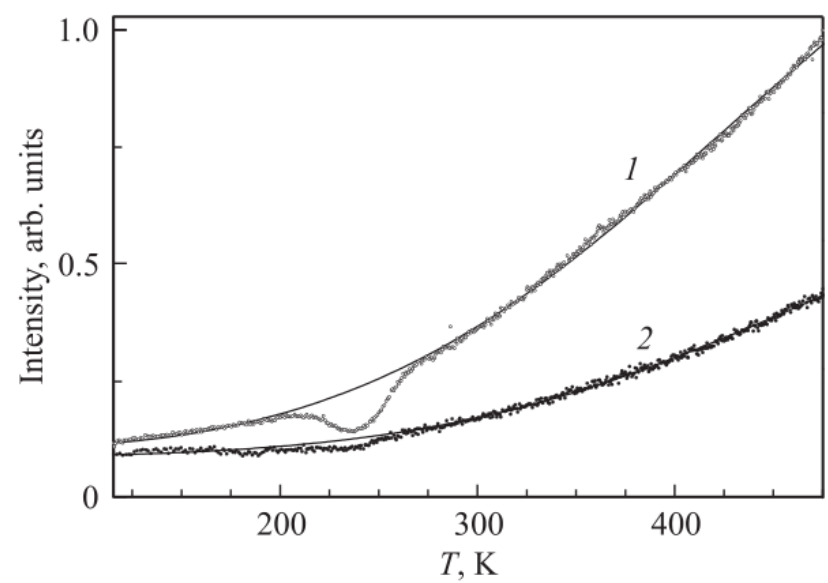

Figure 1 Temperature dependence of the luminescence radiation LGBO: Ce with $\mathrm{E}_{q}=3,97 \mathrm{eV}$, measured under the luminophor radiation with the quantum energy 4,5 eV (1) and 5,0 eV (2). The points are the experimental data, the lines are the approximation results.

As demonstrated on the figure 1, the responsivity of the density of the luminophor emission decreases under increasing of the quantum energy emission. Such a result confirms a previous hypothesis of the insufficiency of the quantum energy emission that leads to creation of the luminescence radiation only high energetic centers of the luminescence, as under increasing of the quantum energy a percent of the luminescence centers for which the equation (1) is not used, decreases. One more confirmation of the validity of this assumption is his full accordance with a statement of Vavilov's Law [6], its mathematics for this case can be noted:

$$
\eta(T)=\frac{N_{L}(T)}{N_{e}},
$$

$\eta(T)$ - temperature depended quantum yield of luminescence; $\mathrm{N}_{\mathrm{e}}$ - number of quantum's exciting radiation on the luminophor; $\mathrm{N}_{\mathrm{L}}(\mathrm{T})$ - number of quantums of the luminescence radiation. 
Such effect of the temperature dependence of the luminescence radiation provides a high sensibility only under a condition of existence of a large number of the low energetic centers of the luminescence that leads to decreasing of the quantum efficiency. Using of the noted effect of the temperature measurement means baseless increasing of the intensity of the exciting radiation for providing of the necessary intensity of the luminescence radiation falling on the photodetector. So, according to above, it may be concluded that using temperature dependence of the luminescence intensity like this has huge limits for usage, because it needs baseless increasing of the energy intensity measurement that is a result of increasing source density of the exciting radiation.

From the point of view of the noncontact temperature measurement more interesting fact is the fact of the temperature extinction of the luminescence radiation under the temperature increasing of the luminophor. In this case dependence of the quantum yield of luminescence from the temperature can be demonstrate with the following equation $[7,11]$ :

$$
\eta(T)=\frac{1}{1+q e^{\frac{-E_{n}}{k T}}}
$$

where $q$-coefficient shows characteristics of the luminescence center; $k$-Boltzmann's constant; $e$ - Euler's constant.

Hence, the depending of the intensity of the luminescence radiation from the temperature can be demonstrated:

$$
I_{L}=\frac{I_{\max }}{1+q e^{\frac{-E_{n}}{k T}}},
$$

where $I_{\max }$ - the maximum possible level of the максимально intensity of the luminescence radiation for the luminophor under the same level of the intensity and the frequency of the exciting radiation according to Boltzmann's Law.

It is obvious from the equation (4) that the temperature decreasing appears only if some critical level of the luminophor temperature is reached, which is less if the transit energy of the luminescence center on the higher energetic level is less. But at the temperature lower than the critical level the equation must hold true:

$$
I_{L} \approx I_{\max }
$$

The conclusion is confirmed by the experimental researches in the papers [7, 11] (figure 2 and figure 3 ).

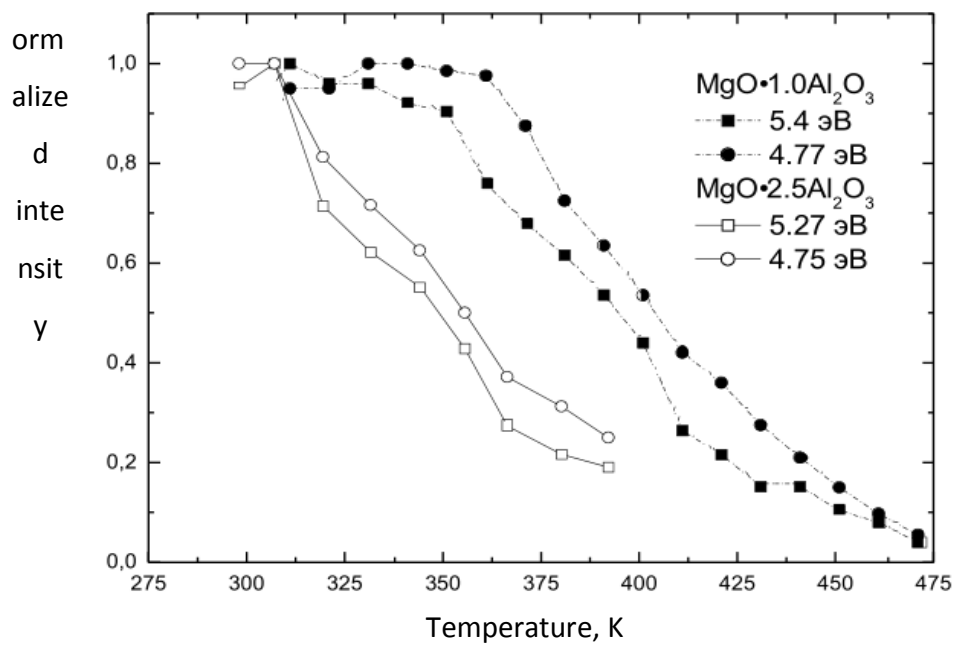

Figure 2 Experimantal dependence of the intensity of the X-rey luminescence on the structural defects of the spinel cristals from the temperature 


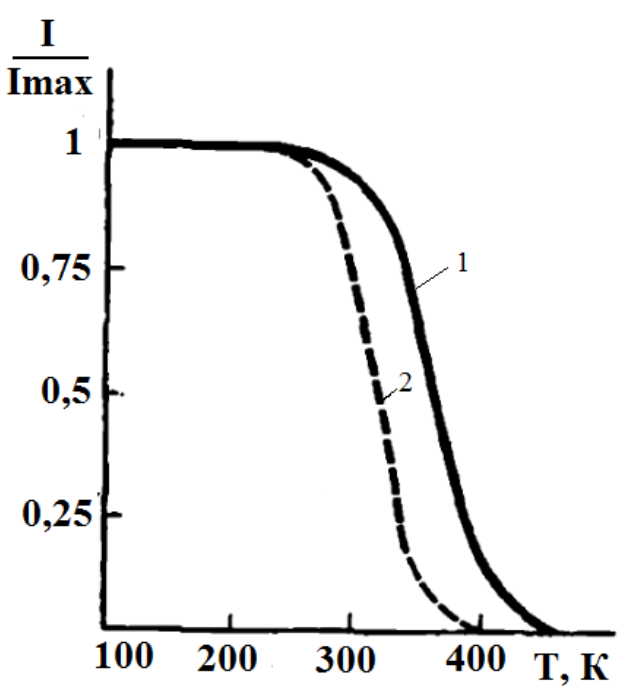

Figure 3 Experimantal dependence of the intensity of the luminescence $\mathrm{ZnS}-1 \cdot 10^{-4} \mathrm{Ag}$, Co- from the temperature, under cobalt concentration $1,8 \cdot 10^{-6}(1)$ and $1,84 \cdot 10^{-4}(2)$

From the figures 2 and 3 it is obvious that the dependence of the intensity of the luminescence radiation from the temperature under the constant intensity of the exciting radiation within really short limits that corresponds to the differential temperature range of the pole winding of the electrical machine, has a quasilinear characteristic, that determines the straight-line relation of the statistic characteristic of the temperature sensor, created on this effect. Herewith, considering (5), we can notice that if the choice of the material- luminophoris right for this type of the luminescence in the area of the high measurement limit the maximum level of the energy efficiency can rise, that allows to avoid baseless increasing of the intensity of the exiting radiation.

Using the noted effect of the temperature decreasing of the luminescence, we determine the main idea of the noncontact method of the temperature measurement that anticipates the thermal equilibration between the research object and the luminescent coating with the following temperature transformation of the luminophor into the intensity of the luminescence radiation with the radiation of the luminescent coating of the exiting radiation. Herewith, the value of the intensity of the luminescence radiation can be transferred into the proportional level of the constant voltage using the photodetector that locates at some distance from the research object. Termination of the measurement will be made with the transformation of the out voltage of the photodetector as the result of the measurement.

For the realization of suggested method of the noncontact temperature measurement there is offered the construction of the primary transducer which structure is shown in figure 4.

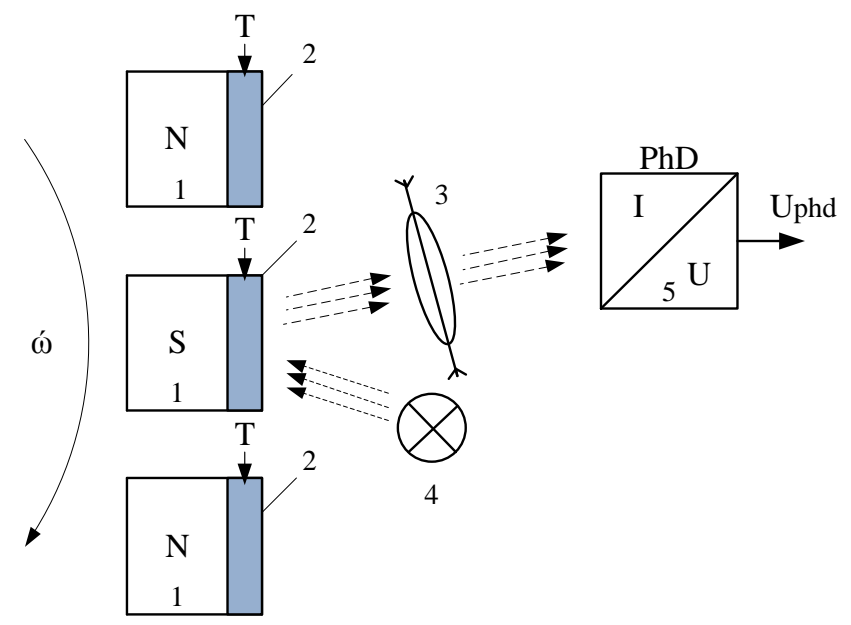

Figure 4 Structure of the noncontact temperature sensor

The action of the noncontact temperature sensor in the figure 4 is performed as follows. On irradiation of the luminescent coating 2 in the thermal balance with the research object 1 (for example, the pole winding of the electrical machine), the stream of the luminescence radiation is created, its capacity, considering (4), is determines: 


$$
P_{L}=\frac{I_{\max } S_{e a}}{1+q e^{\frac{-E_{n}}{k T}}},
$$

where $S_{e f}$ - the effective area of the analyzing luminescent coating.

The luminous flux of the luminophor gets through a filter 3 that flows through itself only a little strip of spectrum of the light emission corresponding with the maximum of the luminescence. As a result there is a decreasing of the influence of the luminophor thermal emission and the research object, As well as the influence of the other sources emission, which can lead to the imprecisions in the result of the measurement. A filtered signal comes on the receiver input 5 , in which the capacity of the luminous flux transforms into the out voltage.

Considering the equation (6), the equation of the transformation of the noncontact temperature sensor will look like:

$$
U_{p h d}=\frac{I_{\max } S_{e a} \alpha \beta \gamma}{1+q e^{\frac{-E_{n}}{k T}}},
$$

where $a$-coefficient of the transmission of the propagation medium of the luminous flux that takes into account the distance between the luminophor and the photodetector and the optical transmission of this environment on a frequency of the maximum of the luminescence radiation; $\beta$ - coefficient of the transmission of the filter; $\gamma$ coefficient of the photodetector transformation.

The simulation result (7) for the luminophor parameters: the largest possible value of the luminescence radiation $-100 \mathrm{Wt} / \mathrm{m}^{2}$, the energy for the transformation of the luminescent center on the higher energy $-0,7 \mathrm{eV}$, the constant, characterizing features of the luminescent center $-10^{9}$, the multiplication of the coefficients of the coefficient of the transmission of the propagation, coefficient of the transmission of the filter and the coefficient of the photodetector transformation $-0,5 \mathrm{Wt} / \mathrm{V}$ on the effective area oa the luminescent coating - $5 \mathrm{~mm}-5$ $\mathrm{mm}^{2}$ in the figure 5 .

From the figure 5 it is obvious that the offered sensor under the noted characteristic provides high enough sensitivity, and its statistic characteristic has monotonically decreasing trend all through the measuring range. Besides, from the figure 5 it is obvious, with the short measuring range of the temperature change (from $360^{\circ} \mathrm{K}$ to $420^{\circ} \mathrm{K}$ ) the statistic sensor characteristic is a line in fact.

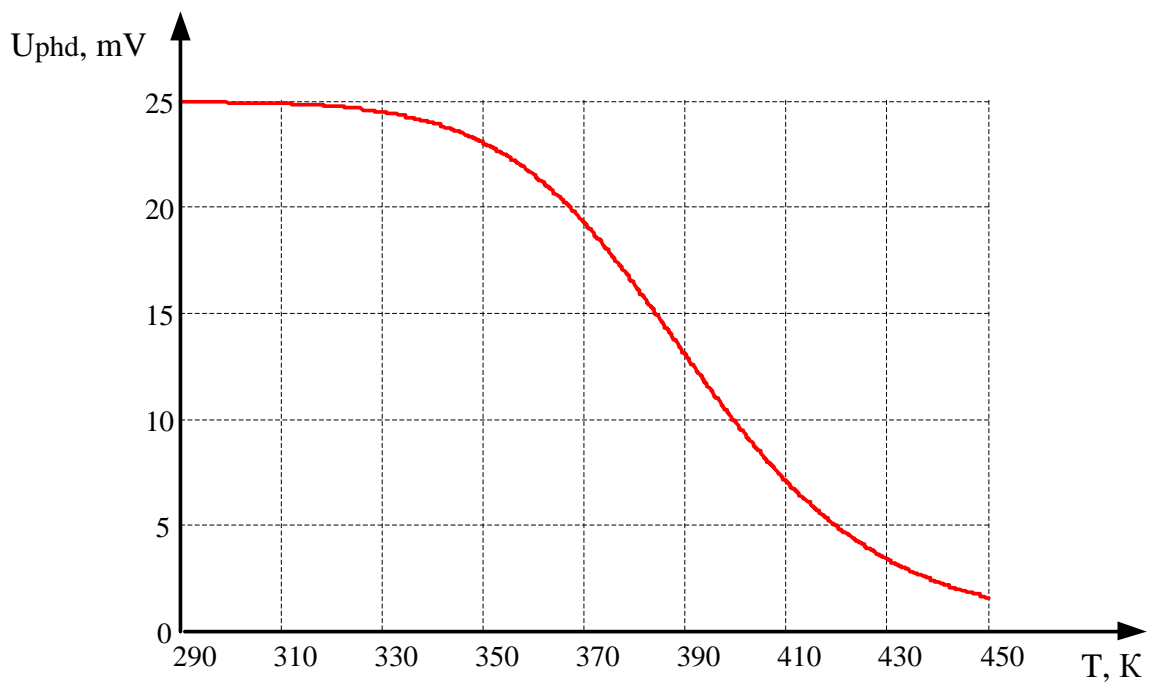

Figure 5 Statistic characteristic of the offered noncontact temperature sensor

\section{EXECUTIVE SUMMARY}

1. Offered new noncontact method of temperature measurement based on the phenomenon of the luminophor temperature decreasing effect allowed to provide a high responsivity and transformation of the informational signal in the area of the higher frequencies, it increased the temperature measurement accuracy compared to the methods of the thermal pyrometry.

2. Developed the construction of the new noncontact temperature sensor using offered new noncontact method of measurement characterized a linear statistic parameter in a short measuring range of the temperature change and a low energy intensity. Theoretically, there was a transfer equation connected the temperature of the research object with the output direction of the developed noncontact sensor. 


\section{REFERENCES}

1. Posudin Y. I. Physics and biophysics of the environment / Y. I. Posudin - Kyiv: Svit, 2000. -303 p.

2. Dubas L. G. Noncontact pyrometry for the condensed material / L. G. Dubas // Magezine of the technic physics. - 2013. - Part 83, n. 1 - P. $134-139$.

3. Hotra O. Z. Microelectronic components and devices for thermometry / O. Z. Hotra - Lviv: LigaPres, $2001-487 \mathrm{p}$.

4. V. V. Kukharchuk Metod analytical calculations vibration in overclocking mode hydrounit / Kukharchuk V. V., Hraniak V. F., Vedmitskyi Y. G. // Bulletin of engineering academy of Ukraine. - 2015. - № 2. - P. 89-93.

5. Belikova L. Y. Electric machines. Tutorial. / L. Y. Belicova, V. P. Shevchenko - Odesa: Nauka i tehnika, 2011. - 480 p.

6. Martynson L. K. Quantum physics. Tutorial. / L. K. Martynson, E. V. Smirnov - Moscow: Publ. MNTU N. E. Buman, 2004. - 496 p.

7. Gurvych A. M. Introduction in physics chemistry of crystallinephosphor. Tutorial for Universities. / A. M. Gurvych - Moscow: «Vysshaya shkola», 1971. - 336 p.

8. Lansberg L. S. Optics / G. S. Lansberg - Moscow: Phismatlit, 2003. - 848 p.

9. Microelectronic Signal transducers of thermal sensors flow. Monograph / Z. Y. Hotra, S. V. Pavlov, R. L. Golyaka and other - Vinnitsa: VSTU, 2012. - 240 p.

10. Ogorodnikov I. N. Luminiscence and electron excitation in crystals $\mathrm{Li}_{6} \mathrm{Gd}\left(\mathrm{BO}_{3}\right)_{3} \wedge \mathrm{Ce}^{3+} / \mathrm{I}$. N. Ogorodnikov, I. N. Sedunova, L. I. Isaenko, S. A. Zhurkov // Physics of the solid body . - 2013. part 54, n. 3 - P. $457-464$.

11. Kazarynov Y. G. Luminescent characteristics of the spinel monocrystal on exposure to ionization radiation / Y. G. Kazarynov, M. T. Gritsyna, V. A. Kobyakov, K. E. Sikafus // Issues of the nuclear science and technics - 2002. - №3. - P. 53 - 57. 